\title{
Assimetria matéria-antimatéria - a violação de CP em interações fortes e fracas
}

\author{
Matter-antimatter asymmetry - CP violation in strong and weak interactions \\ Lucas Falcão ${ }^{* 10}$ \\ ${ }^{1}$ Centro Brasileiro de Pesquisas Físicas, CEP: 22290-180, Rio de Janeiro, RJ, Brasil.
}

\begin{abstract}
Recebido em 23 de agosto de 2021. Revisado em 18 de novembro de 2021. Aceito em 24 de novembro de 2021.
O objetivo deste trabalho é a descrição teórica do fenômeno de violação de CP nas interações fortes e fracas. Para as interações fracas, veremos em quais ocasiões a violação de CP foi observada e como ela é prevista pelo Modelo Padrão, segundo a matriz CKM. No caso das interações fortes, é descrito como a estrutura do vácuo na QCD, necessária para resolver o problema $U(1)_{A}$, prediz a presença de um termo proporcional à $\theta$ que viola CP. Para concordar com os limites experimentais esse parâmetro deve ser pequeno $\left(\theta \leq 10^{-10}\right)$. O foco é a solução quiral proposta por Peccei e Quinn, que esta relacionada à uma partícula pseudoescalar leve, o áxion.
\end{abstract}

Palavras-chave: Assimetria matéria-antimatéria, Violação de CP.

\begin{abstract}
The aim of this work is the theoretical description of $\mathrm{CP}$ violation phenomena in strong and weak interactions For weak interactions, we will see the occasions when $\mathrm{CP}$ violations were observed and how this is predicted by the Standard Model, according to the CKM matrix. In the case of strong interactions, it is described how the vacuum structure in $\mathrm{QCD}$, necessary to solve the problem $U(1)_{A}$, predicts the presence of a term proportional to $\theta$ that violates CP. To agree with the experimental limits this parameter must be small $\left(\theta \leq 10^{-10}\right)$. The focus is on the chiral solution proposed by Peccei and Quinn, which is related to a light pseudoscalar particle, the axion. Keywords: Matter-antimatter asymmetry, $\mathrm{CP}$ violation.
\end{abstract}

\section{Introdução}

No começo do século 20, os átomos ainda não eram muito bem compreendidos, sabíamos que tudo era feito de matéria mas não entendíamos muito sua natureza. Por volta do final da década de 20, Dirac tentava unir dois conceitos importantes para física: a relatividade e a mecânica quântica. Sua famosa equação possuía uma estranha solução que sugeria a existência de dois estados para o "elétron", um como já conhecemos - o próprio elétron - e outro exatamente igual ao elétron porém com carga oposta; esta partícula seria nomeada de pósitron. Este fato abrira portas para um dos maiores mistérios da física: porque o universo é feito apenas desta matéria que conhecemos, e não também de antimatéria? A isso damos o nome de assimetria matéria-antimatéria.

Aqui introduzimos a violação de carga-paridade (VCP), uma das condições necessárias para explicarmos a assimetria de matéria-antimatéria do universo, mas não a única [1]. Trata-se de uma combinação das simetrias da conjugação de carga $\mathrm{C}$, que consiste em trocar uma partícula por sua antipartícula, ou seja, todos os números quânticos aditivos da partícula são invertidos, e a paridade $\mathrm{P}$, que inverte o sinal das coordenadas espaciais.

\footnotetext{
*Endereço de correspondência: lucas-falcao@cbpf.br
}

Como já é conhecido, o universo do cotidiano é composto de partículas. Praticamente todos os fenômenos físicos das partículas conhecidas são bem descritos pelo Modelo Padrão (MP) das partículas elementares e suas interações fundamentais (para saber mais sobre MP veja [2]). O MP fornece um arcabouço teórico muito elegante e, apesar de possuir limitações, passou com sucesso por testes precisos 3 .

O Modelo Padrão inclui VCP através de uma fase complexa na chamada matriz de mistura de quarks Cabibbo-Kobayashi-Maskawa (CKM). Apesar de pequena, a observação da violação de CP foi estabelecida em interações fracas nos sistemas de mésons $K$ e $B$ por vários experimentos e, mais recentemente, no setor charmoso, em canais neutros [4] ${ }^{1}$

Já para as interações fortes, apesar de ainda não existir comprovação experimental, existem possibilidades teóricas de violação de CP. No início dos anos de 1970 existia um problema que se tornou mais evidente com o desenvolvimento da QCD. A lagrangeana da QCD para $\mathrm{N}$ sabores, no limite de quarks sem massa, possuía uma simetria global: $U(N)_{V} \times U(N)_{A}$, onde por sabores se quer dizer os tipos diferentes de quarks. Como $m_{u}, m_{d}<<\Lambda_{Q C D}$, sendo $m_{u}, m_{d}$ as massas dos quarks up e down e $\Lambda_{Q C D}$ uma escala de energia onde as contribuições não perturbativas se tornam dominantes,

\footnotetext{
${ }^{1}$ Parte deste trecho está escrito com mais detalhes em 5
} 
pelo menos para esses quarks seria sensível, ou seja, poderíamos considerar $U(2)_{V} \times U(2)_{A}$, invariante.

$\mathrm{O}$ que se encontrava experimentalmente é que o vetor de simetria correspondente ao isopin vezes número barônico $\left(U(2)_{V}=S U(2)_{I} \times U(1)_{B}\right)$ seria uma boa simetria da natureza. Para simetrias axiais não seria bem assim. Condensados de quarks quebravam simetrias axiais espontaneamente, para o caso de $U(1)_{A}$. Não era esperada a mistura de paridade nos multipletos no espectro hadrônico, mas sim vestígios de bósons de Goldstone associado a quebra em $U(2)$, sendo $U(2)_{A}=$ $S U(2)_{A} \times U(1)_{A}$.

Weinberg apelidou este problema de $U(1)_{A}$ e sugeriu que de alguma forma não existiria simetria $U(1)_{A}$ em interações fortes, o problema consistia em não existir um bóson de Goldstone correspondente a está quebra de simetria.

A solução para o problema de $U(1)_{A}$ veio com 't Hooft, propondo uma estrutura de vácuo mais complexa para QCD. Essa maior complexidade fez com que $U(1)_{A}$ não fosse uma verdadeira simetria da $\mathrm{QCD}$, mesmo que pareça ser uma simetria na lagrangeana da QCD no limite de quarks sem massa.

Junto a essa estrutura de vácuo complicada surgiu um parâmetro de fase $\theta$ que, devido à sua forte relação com o momento dipolar do nêutron, deveria ser muito pequeno mas não nulo, e apenas caso $\theta=0$ que a $\mathrm{CP}$ não seria violada nas interações fortes.

Então a solução para o problema $U(1)_{A}$ criou um novo problema: Por que $\theta$ é tão pequeno?.

Uma possível solução para o problema $U(1)_{A}$ parece ser fornecermos uma anomalia quiral (uma anomalia se trata de uma situação com invariância ao nível clássico, mas sem simetria ao nivel quântico, para melhores explicações sobre anomalia quiral veja [6]) para correntes axiais. A divergência da corrente axial $J_{5}^{\mu}$ associada a $U(1)_{A}$ pega correções quânticas do diagrama de Feynamn que conecta os dois campos e glúons com os quarks. Essa anomalia fornece um valor diferente de 0 para $J_{5}^{\mu}$.

Este artigo é organizado na seguinte forma:

- Seção 2: são apresentados conceitos básicos do Modelo Padrão e das simetrias CPT, bem como a primeira observação de VCP.

- Seção 3: aqui é aprofundado o problema da violação de CP nas interações fortes e suas possíveis soluções.

- Seção 4: nesta são apontadas questões sobre a violação de CP nas interações fracas e onde já foram observadas experimentalmente.

\section{Fundamentos Teóricos}

\subsection{O Modelo Padrão}

As chamadas partículas elementares são os constituintes menores da matéria, sem subestrutura conhecida até os limites atuais de $10^{-19}$ metros ou menos. As separamos em dois tipos: o bloco básico de construção da matéria, e o bloco responsável por intermediar a interação do primeiro. O primeiro bloco são os férmions, partículas de spin semi-inteiro que são subdivididas em quarks e léptons. As demais partículas são os bósons mediadores das interações fundamentais, que podem ser separados em escalar ou vetorial, dependendo de seu spin. Segundo o Modelo Padrão, os férmions possuem spin $1 / 2$ e os bósons spin 0 ou 1, e as partículas interagem através das interações eletromagnética, fraca e forte, a depender de características da própria partícula (interações gravitacionais não estão no escopo do MP). A Figura 1 mostra as partículas elementares do MP.

- Fóton $(\gamma)$ : responsável por mediar a interação eletromagnética, não possui massa e nem carga elétrica. Por ter spin 1 se trata de um bóson vetorial.

- $W^{ \pm}$e $Z^{0}$ : responsáveis por mediar as interações fracas, carregadas e neutras respectivamente; são bósons massivos e também vetoriais.

- Glúons $(g)$ : responsáveis por mediar as interações fortes, também não possuem massa mas possuem carga cor, que é um número quântico intríseco de partículas que interagem fortemente. Glúons interagem entre si e são vetoriais.

- Higgs: responsável por mediar a interação das partículas com campo de Higgs, fornecendo massa às mesmas. É o único bóson escalar da teoria e possui massa.

Os Diagramas de Feynman da Figura 2 mostram os vértices básicos das interações dentro do Modelo Padrão. As interações eletromagnéticas, fortes e fracas neutras preservam sabor, enquanto que interações fracas carregadas promovem mudança de sabor, como por

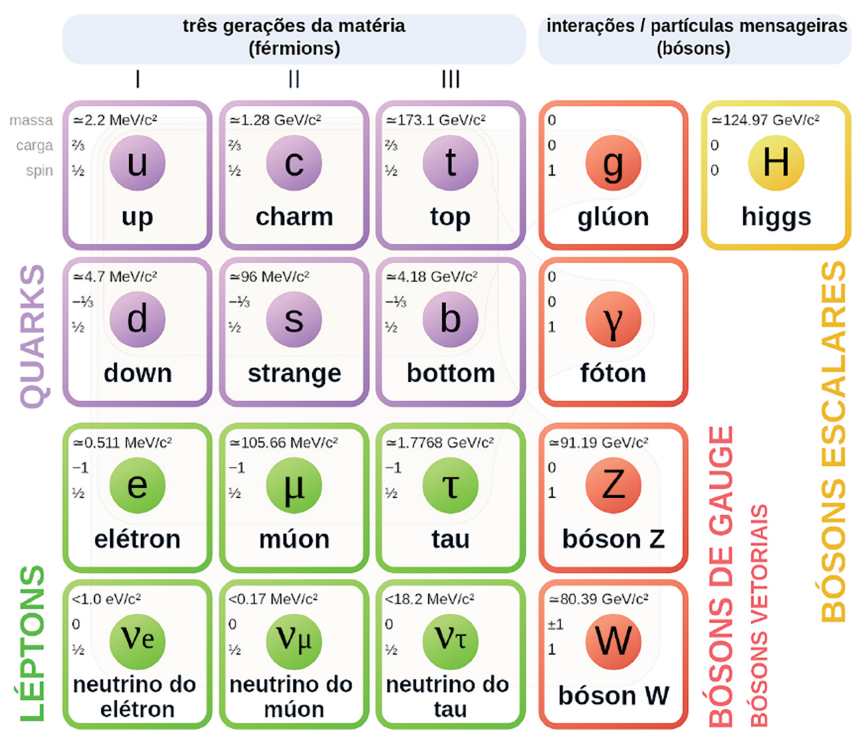

Figura 1: Modelo Padrão de Párticulas Elementares [7]. 

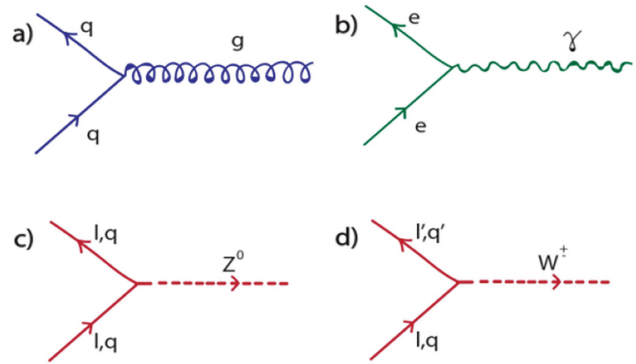

Figura 2: Diagramas de Feynman dos vértices básicos das interações fundamentais: a) interação forte que atua nos quarks, b) interação eletromagnética que atua em partículas carregadas eletricamente, c) e d) interação fraca que atua nos léptons e quarks, d) é a única que troca o sabor das partículas.

exemplo no decaimento de um quark $c$ em um quark $s$ através de um bóson $W^{+}$.

Por conta das propriedades das interações fortes, os quarks não são encontrados individualmente na natureza, senão em estados ligados, os chamados hádrons que podem ser divididos em bárions e mésons:

- Bárions: partículas formadas por 3 quarks (ou antiquarks), como os prótons (uud) e nêutrons $(u d d)$. Por terem spin semi-inteiro são férmions.

- Mésons: partículas formadas por um par quarkantiquark, como o píon $(u \bar{d})$. Por terem spin inteiro são bósons.

O ambiente das teorias quânticas de campos é adequado para contemplar as interações fundamentais do Modelo Padrão. Este pode ser visto como a união de algumas delas: a quantum electrodynamics (QED), que trata sobre as interações eletromagnéticas; a quantum chromodynamics (QCD), que trata sobre as interações fortes; e a quantum flavour dynamics (QFD), que trata sobre as interações fracas.

De um ponto de vista mais técnico, o MP se trata de uma teoria de calibre, não abeliana, com quebra espôntanea de simetria (quando as equações de movimento são invariantes, mas o estado fundamental não é, para mais informações sobre QES veja [8]). Enquanto teoria quântica de campos renormalizável é baseada na simetria de calibre $S U(3)_{C} \times S U(2)_{L} \times U(1)_{Y}$. Nesse grupo de simetrias, $S U(3)_{C}$ trata das interações fortes, e $S U(2)_{L} \times U(1)_{Y}$ das interações eletrofracas, antes da QES. Após a QES, devido à interação com o campo de Higgs, os bósons das $W^{ \pm}$e $Z^{0}$ adquirem massa - além de todos os férmions - e o fotón permanece não massivo.

Então, podemos escrever a lagrangeana básica do Modelo Padrão como uma composição desses grupos de simetria, mais uma parte referente ao mecanismo de Higgs:

$$
\mathcal{L}_{S M}=\mathcal{L} S U(3)_{c}+\mathcal{L}\left(S U(2)_{L} \times U(1)_{Y}\right)+\mathcal{L}_{\text {Higgs }}
$$

Podemos escrevê-la em seu formato mais popular, com cada termo responsável por um setor (para mais informações veja [9] e [10]):

$$
\begin{array}{rr}
\mathcal{L}=-\frac{1}{4} F_{\mu \nu} F^{\mu \nu} & \text { (Partículas de interação) } \\
+i \bar{\Psi} D \Psi+\text { h.c. } & \text { (Interação entre partículas de matéria) } \\
+\Psi_{i} y_{i j} \Psi_{j} \phi+\text { h.c. } & \text { (Massa para partículas de matéria) } \\
+\left|D_{\mu} \phi\right|^{2} & \text { (Massa para partículas de interação) } \\
-V(\phi) & \text { (Auto-interação do Higgs) }
\end{array}
$$

\subsection{Simetrias CPT}

Por volta do final da década de 40, pelas mãos de Feynman, Schwinger e Tomonaga, a QED renormalizável viria a se tornar uma espécie de paradigma. Por ser invariante sob as simetrias de $\mathrm{C}, \mathrm{P}$ e $\mathrm{T}$, e dada sua relação com a invariância de Lorentz, admitiu-se que as outras interações também conservariam $\mathrm{C}, \mathrm{P}$ e $\mathrm{T}$. Vejamos do que se tratam essas três operações:

- C: a operação de conjugação de carga inverte todos os números quânticos aditivos, como o número bariônico, leptônico e carga elétrica. É a operação que troca partícula por antipartícula. Dizemos que um estado é autoestado de $\mathrm{C}$ caso não seja alterado por ele, como é o caso, por exemplo, de alguns mésons neutros e o fóton.

- P: a conjugação de paridade promove a inversão das coordenadas espaciais em relação à origem. A aplicação do operador $\mathrm{P}$ em vetores comuns acontece da seguinte forma: $\vec{r} \rightarrow-\vec{r}$ e $\vec{p} \rightarrow-\vec{p}$. Porém, por exemplo, para o caso de um pseudovetor como é o momento angular, não existe mudança de sinal. Assim sendo, o spin também não é afetado pelo operador $\mathrm{P}$, e esta característica é importante para o que virá a seguir.

- T: o operador de reversão temporal faz a transformação discreta $t \rightarrow-t$. Ao aplicarmos um operador $\mathbf{T}$ em uma função de onda $\psi$, obtemos $\psi^{*}$, sendo $\psi^{*}$ o complexo conjugado de $\psi$. Isto é o equivalente a mudar o sinal do vetor velocidade sem alterar a posição e, portanto, a operação pode ser entendida como uma reversão de movimento. Na mecânica quântica não relativística, caso a hamiltoniana seja real, a reversão temporal conserva a equação de Schrödinger invariante sob $\mathrm{T}$.

\subsection{Primeiras observações de violação}

Até meados da década de 50, se imaginava que todas as interações fundamentais fossem invariantes sob essas simetrias. Em 1956, Yang e Lee publicaram um artigo que questionava a conservação de paridade nas interações fracas [11. Em seus estudos, eles observaram que não existia evidência de que esta simetria deveria ser convervada neste tipo de interação. Este foi o pontapé inicial para que Madame Wu, em 1957, iniciasse seu trabalho com decaimentos $\beta$ de Cobalto-60 [12]. Seu experimento se tratava da observação do decaimento de núcleos de 
Cobalto-60, com spin alinhado por um campo magnético intenso, em Níquel-60, via força fraca, produzindo um elétron e um antineutrino do elétron. Como o spin deve ser conservado após a interação, e, caso as interações fracas conservassem paridade, as partículas produzidas após o decaimento deveriam ser emitidas em quantidades iguais para cima e para baixo. Ou seja, o caso em que o elétron era emitido para cima e, por conservação de momentum, o neutrino era emitido para baixo, deveria acontecer tantas vezes quanto o contrário. Porém, ela observava que existia uma direção preferencial para o elétron, ele sempre era emitido em direção oposta ao spin do núcleo de cobalto. Este experimento é especial pois demonstra a preferência das interações fracas por certas configurações de spin, o que viola completamente a simetria de paridade.

Tais observações de Madame Wu estão relacionadas ao caráter quiral das interações fracas, elas distinguem partículas e antipartículas de acordo com a sua quiralidade. De forma semelhante, Yang, Lee e Oeheme questionaram também a conservação de $\mathrm{C}$ nas interações fracas, ainda em 1957 [13], demonstrando que esta simetria não se mantinha em decaimentos leptônicos do píon. E por último, mais recentemente foi observada a violação de simetria $\mathrm{T}$ em mésons $\mathrm{B}$ neutros pelo experimento BaBar 14.

Uma vez que as simetrias $\mathrm{C}$ e $\mathrm{P}$ eram violadas separadamente nas interações fracas, a aplicação de ambas juntas era esperada ser uma boa simetria, visto que só eram observados neutrinos de helicidade de mão esquerda e anti-neutrinos de helicidade de mão direita. Esta combinação de simetrias foi colocada à prova em 1964, por James Cronin e Val Fitch [15]. Seu trabalho envolvia a observação do decaimento de um feixe de mésons $K$ neutros, que podem ser subdivididos em $K_{S}$ e $K_{L}$, onde S (de short) e L (de long) faz referência as suas vidas médias, mais curta e mais longa, respectivamente. Esta divisão está relacionada com os principais canais hadrônicos de decaimento dos káons, em 2 ou 3 píons, respectivamente. Se acreditava que o $K_{S}$, com tempo de vida menor, deveria decair somente em 2 píons, que tem autovalor de $\mathrm{CP}$ igual a +1 , enquanto o $K_{L}$ iria a 3 píons, com autovalor de CP igual a -1 . Cronin e Fitch observavam que eventualmente o $K_{L}$ podia decair em 2 píons, o que era uma evidência de violação de CP. Finalmente, podemos falar sobre a combinação das 3 simetrias. O teorema CPT sustenta a idéia de que qualquer teoria quântica de campos unitária, local, com invariância de Lorentz e causalidade no espaço de Minkowski, é invariante sob CPT 16. Atualmente o teorema CPT se mantém como uma simetria exata para todas as interações e não há indício experimental do contrário.

Uma vez que já foram vistos os fundamentos teóricos e já foi feito um resgate histórico, vejamos as peculiaridades de cada uma das interações que interessam a este trabalho.

\section{O problema da CP forte}

O Modelo Padrão, apesar de eficiente e bem-sucedido em diversos testes, possui alguns problemas, sendo um deles o problema da CP forte. Este problema envolve o parâmetro $\theta$, que está associado à um termo na lagrangeana da QCD.

$$
\mathcal{L}=\theta \frac{g^{2}}{16 \pi^{2}} F_{\mu \nu}^{a} \widetilde{F}^{\mu \nu a}
$$

onde

$$
\widetilde{F}_{\mu \nu \rho \sigma}^{a}=\frac{1}{2} \epsilon_{\mu \nu \rho \sigma} F^{\rho \sigma a}
$$

Este termo respeita as simetrias da QCD, mas viola CP [17, e pode contribuir para o momento de dipolo elétrico do nêutron $(\mathrm{eDMN}), d_{n}$. Isto fica claro ao avaliarmos o caso da QED, por ser proporcional à $\vec{E} \cdot \vec{B}$, e como o vetor campo elétrico troca de sinal sob o operador de paridade mas o campo magnético não, $\vec{E} \stackrel{P}{\rightarrow}-\vec{E}, \vec{B} \stackrel{P}{\rightarrow} \vec{B}$, ou seja é ímpar sob P. Como parte da lagrangeana é $\vec{E}^{2}-\overrightarrow{B^{2}}$, que é par sob paridade, $\mathrm{P}$ é violada se estes termos existissem na lagrangeana, Para uma teoria Abeliana, o termo ímpar é uma 4-divergência e não modifica as equações de movimento, isto não é possível para uma teoria não-Abeliana como a QCD.

\subsection{Momento de dipolo elétrico do nêutron - eDMN}

No coração do problema de CP forte está a questão do porquê o momento de dipolo elétrico do nêutron é tão pequeno, este é proportional ao $\theta$. O conceito de momento de dipolo elétrico é simples: duas cargas elétricas, com cargas opostas, separadas por uma distância, calculado convencionalmente como $\vec{d}=q \vec{r}$. Através de cálculos usando correções quânticas é possível encontrarmos um valor aproximado para o $d_{n}[18$ :

$$
d_{n}=\left(5,2 \times 10^{-16} \text { e cm }\right) \theta
$$

Como o eDMN é um vetor, ele precisa apontar em alguma direção. $\mathrm{O}$ nêutron tem apenas um único vetor que quebra a simetria de Lorentz, que é o spin. Portanto, o eDMN deve apontar na mesma direção que o spin.

Alguns experimentos tentaram medir o eDMN e a maneira mais simples é via precessão. Imagine uma situação em que uma porção de spins up estão apontando numa mesma direção. Quando aplicado um campo elétrico e magnético paralelamente ao sistema, teremos a precessão de Larmor [19]:

$$
\nu_{ \pm}=2|\mu B \pm d E|
$$

Onde $\nu$ é a frequência de precessão, $\mu$ e $d$ os momentos dipolo magnéticos e elétricos dos nêutrons, respectivamente. Ambos são paralelos ou antiparalelos ao spin do nêutron. A mudança na frequência de precessão sinaliza 
um valor diferente de zero do eDMN quando o campo elétrico é aplicado paralelamente e antiparalelo ao campo magnético. A mudança de frequência é medida como a diferença de fase da polarização do nêutron após a precessão do spin por um tempo fixo $t$. Uma vez que o campo externo é ligado, depois de algum tempo, ao desligarmos os campos, podemos medir quantos dos nêutrons mudaram pra posição spin down. Assim temos a medida de frequência $\nu_{+}$. Fazendo novamente o experimento só que com campos anti-paralelos podemos medir $\nu_{-}$. Tomando a diferença entre essas duas frequências o eDMN pode ser balizado.

$$
\left|d_{n}\right| \leq 10^{-26} \text { e } \mathrm{cm}
$$

Comparando as equações 3 e 5 se apresenta o problema, por que $\theta \leq 10^{-10}$ ?

\section{2. Áxions}

\subsection{1. Áxion como solução para CP forte}

A explicação mais aceita para a pequenez de $\theta$ envolve a hipótese de uma nova partícula, o áxion. O áxion foi postulado por Peccei e Quinn em 1977 para resolver o problema da CP forte na QCD. A idéia de Peccei e Quinn era fazer de $\theta$ um campo, o que faz com que surja um nova simetria global que será espontaneamente quebrável.

Eles apresentam $\theta$ como um compoenente funcional, a simetria global U(1) sob a qual um campo complexo é carregado. Essa simetria é quebrada espontaneamente pelo valor esperado para o vácuo (VEV), obtido por esse campo escalar. O áxion é o bóson de Goldstone dessa quebra de simetria.

Ou seja, ao introduzirmos uma simetria quiral global $\mathrm{U}(1)$ - que depois será conhecida como $U(1)_{P Q}-$ fornecemos o que parece ser a solução mais aceitável para o problema de CP forte. Como essa simetria é espontaneamente quebrada, ao introduzirmos na teoria geral, substituímos uma violação de CP estática por uma violação de $\mathrm{CP}$ dinâmica, com o áxion.

O que precisamos é de um campo escalar $(\phi)$ que acopla a um campo de quark $(q) . U(1)_{P Q}$ precisa ser quiral para termos uma anomalia. Por exemplo, escolhemos que a transformação afete apenas as componentes de mão direita dos campos de quarks, neste caso a simetria será:

$$
q_{R} \rightarrow e^{-i \alpha} q_{R} \quad \phi \rightarrow e^{i \alpha} \phi
$$

Como resultado dessa transformação, o campo de áxions se desloca:

$$
a(x) \rightarrow a(x)+\alpha f_{a}
$$

Uma vez que $\frac{a}{f_{a}}$ é uma fase do campo escalar, $U(1)_{P Q}$ atua sobre ele como na Eq. 7. onde $f_{a}$ é conhecido como constante de decaimento do áxion. Para fazermos a lagrangeana do Modelo Padrão invariante sob $U(1)_{P Q}$ devemos considerar agora as interações com os áxions, teremos:

$$
\begin{aligned}
L_{T O T A L}= & L_{M P}+\theta \frac{g^{2}}{16 \pi^{2}} F_{\mu \nu}^{a} \widetilde{F}^{\mu \nu a}-\frac{1}{2} \partial_{\mu} a \partial^{\mu} a \\
& +L_{i n t}\left[\partial^{\mu} a / f_{a}\right]+\xi \frac{a}{f_{a}} \frac{g^{2}}{32 \pi^{2}} F_{a}^{\mu \nu} \widetilde{F}_{a \mu \nu}
\end{aligned}
$$

onde $\xi$ depende do modelo de áxion específico. O último termo é necessário para assegurar que $U(1)_{P Q}$ tem de fato uma anomalia:

$$
\partial_{\mu} j_{P Q}^{\mu}=\xi \frac{g^{2}}{32 \pi^{2}} F_{a}^{\mu \nu} \widetilde{F}_{a \mu \nu}
$$

Este termo representa um potencial efetivo pro campo de axions, seu mínimo ocorre em $<a>=\frac{f_{a}}{\xi} \theta$. Um potencial efetivo se trata do valor mínimo esperado do operador hamiltoniano calculado no estado [8]. Um exemplo para compreender o que é um potencial efetivo são os elétrons de um átomo. Eles são distribuídos em níveis de energia diferentes. Calcular o potencial verdadeiro deste sistema é complicado, por conta disso é usado o potencial efetivo, que seria uma espécie de potencial aproximado que descreveria a interação do sistema de uma forma simples, sem levar em conta as propriedades intrínsecas de cada componente do sistema.

Voltando ao áxion, este relaxa até o mínimo de seu potencial. Como estamos no mínimo, o termo de $\theta$ é cancelado, isso fornece uma solução dinâmica ao problema de CP forte. Assim, uma vez que o áxion relaxa até seu mínimo, ele define dinamicamente o eDM de nêutrons para zero. Como afirmado, o áxion QCD resolve o problema de $\mathrm{CP}$ forte.

Não é tão difícil entender a física por trás da solução de Peccei e Quinn. A simetria quiral $U(1)_{P Q}$ permite todos os valores para $\langle a\rangle$ : $0 \leq\langle a\rangle \leq 2 \pi$

\subsubsection{Algumas questões sobre os áxions}

- O áxion já devia ter sido observado?

Vemos os áxions a partir do seu acomplamento com a matéria, esse acomplamento é suprimido por potencias de $f_{a}$. Logo, se $f_{a}$ for grande é dificil de ver o áxion. Além do que, a massa do áxion é proporcional à $f_{a}$ e, como $m_{a} \sim 10^{-5}-10^{-3} \mathrm{eV}$, logo $f_{a} \sim 10^{-12}-10^{-8}$, ou seja, bem pequeno o que ajudaria a explicar a dificuldade de observação.

- Áxions são constituintes da matéria escura?

Pode ter existido uma grande produção de áxions no começo do universo. Como resultado temos um limitador para $f_{a}$. Neste caso, o interessante é que com tais valores para os parâmetros do áxion, impostos pela fenomenologia de partículas, eles podem ser um dos constituintes da máteria escura.

- Como poderiamos encontrar um axion experimentalmente?

$\mathrm{O}$ áxion pode ser acoplado a $F \widetilde{F}$ tanto na QCD quanto na QED. Isso significa que um campo 
magnético muito forte pode converter áxions em fótons. Esse experimento é feito em Livermore e Kyoto. Usando uma cavidade ressonante supercondutora em um campo magnético muito intenso veríamos os áxions em excitações da cavidade.

\section{A violação de $\mathrm{CP}$ nas interações fracas}

\subsection{A primeira matriz de mistura dos quarks}

Para o caso das interações fracas já existem observações bem consolidadas de violação de $\mathrm{CP}$, que está intimamente relacionada à mudança de sabores. A física de sabores trata dos constituintes da matéria, separados em 3 famílias de quarks e léptons, que são distinguíveis perante as interações fracas, de acordo com seu sabor. Essas três gerações possuem iguais acoplamentos de calibre, porém com diferentes massas e interações de Yukawa, isto faz com que existam efeitos de violação de CP e mudança sabor entre as famílias.

Aqui usamos o conceito de estranheza para explicar a primeira matriz de mistura dos quarks, que foi proposta em 1964, conhecida como matriz de Cabibbo. Estranheza é uma propriedade da matéria que algumas partículas possuem, como a carga elétrica, para saber mais sobre estranheza e outras propriedades veja [20]. $\mathrm{Na}$ época, foi comprovado experimentalmente que decaimentos de hádrons que conservavam estranheza possuiam razões de ramificação diferentes dos que não a conservavam. Isto queria dizer que não haveria uma universalidade expressada por uma mesma constante de acoplamento para as interações fracas com $\Delta S=0$ e $\Delta S \neq 0$.

A proposta de Cabibbo envolvia a reobtenção da universalidade das interações fracas, de forma a incluir tanto processos que conservavam estranheza como o contrário. Por conta dessa universalidade, Cabibbo sugeria que processos que conservam estranheza vinham acompanhados de $\cos \left(\theta_{c}\right)$, enquanto o contrário $\sin \left(\theta_{c}\right)$, onde $\theta_{c} \approx 13^{\circ}$ é o ângulo de Cabibbo, obtido experimentalmente. Logo após a teoria de Cabibbo, GellMann e Zweig propuseram a existência de quarks, e todos os hádrons foram entendidos como compostos por combinações de Up, Down e Strange. Em 1970, devido à falta de observação de mudança de sabor em correntes fracas neutras com variação de estranheza (FCNC), Glashow, Iliopoulos e Maiani, propuseram o que viria a ser conhecido como mecanismo GIM [21, que predizia a existência do quark Charm.

Portanto, já no contexto dos quarks, a proposta era de que as cargas fracas se acoplavam, por exemplo, em $u$ e $d^{\prime}$, em vez de $u$ e $d$, onde $d^{\prime}$ é uma combinação linear de $d$ e $s$ :

$$
\begin{aligned}
& d^{\prime}=d \cos \left(\theta_{c}\right)+s \sin \left(\theta_{c}\right) \\
& s^{\prime}=-d \sin \left(\theta_{c}\right)+s \cos \left(\theta_{c}\right)
\end{aligned}
$$

Logo, podemos definir a matriz Cabibbo de mistura das duas primeiras gerações de quarks como:

$$
\left(\begin{array}{l}
d^{\prime} \\
s^{\prime}
\end{array}\right)=\left(\begin{array}{cc}
\cos \theta_{c} & \sin \theta_{c} \\
-\sin \theta_{c} & \cos \theta_{c}
\end{array}\right)\left(\begin{array}{l}
d \\
s
\end{array}\right)
$$

e os dubletos fracos como:

$$
\left(\begin{array}{c}
u \\
d^{\prime}
\end{array}\right) \quad e \quad\left(\begin{array}{c}
c \\
s^{\prime}
\end{array}\right)
$$

Por fim, considerando as interações fracas como uma teoria renormalizável, Maskawa e Kobayashi concluíram, em 1973, que não poderia existir um modelo realista de violação de CP, que havia sido observado em 1964, sem a introdução de uma terceira família de quarks [22. A partir disso é definida a matriz de Cabibbo-KobayashiMaskawa (CKM).

\subsection{A matriz CKM}

A violação de $\mathrm{CP}$ surge a partir da associação dos autoestados de massa $(d, s, b)$ com os autoestados de interação fraca $\left(d^{\prime}, s^{\prime}, b^{\prime}\right)$. Esta associação está contida na matriz CKM, onde cada elemento mede a amplitude de probabilidade de tal transição entre dois quarks ocorrer, Figura 3 .

Vemos na Eq. 14 que a matriz como se ilustra é composta por elementos $V_{i j}$, onde $i=u, c, t$ e $j=d, s, b$. Uma matriz unitária real $3 \times 3$ qualquer possui três graus de liberdade, que seriam os três ângulos de rotação. No caso da CKM, por ser uma matriz complexa, existe mais liberdade na estrutura de fases dos autoestados de massa dos quarks [3. Isto significa que essa liberdade pode ser usada para remover 5 das 6 fases, sobrando então apenas 4 parâmetros independentes: 3 ângulos e uma fase. A matriz CKM é dada por:

$$
\left(\begin{array}{l}
d^{\prime} \\
s^{\prime} \\
b^{\prime}
\end{array}\right)=\left(\begin{array}{lll}
V_{u d} & V_{u s} & V_{u b} \\
V_{c d} & V_{c s} & V_{c b} \\
V_{t d} & V_{t s} & V_{t b}
\end{array}\right)\left(\begin{array}{l}
d \\
s \\
b
\end{array}\right) \equiv V_{C K M}\left(\begin{array}{l}
d \\
s \\
b
\end{array}\right)
$$

Escrita em termos dos três ângulos de mistura e uma fase complexa, ele pode ser apresentada como:

$$
V_{C K M}=\left(\begin{array}{ccc}
c_{12} c_{13} & s_{12} c_{13} & s_{13} e^{-i \delta} \\
-s_{12} c_{23}-c_{12} s_{23} s_{13} e^{i \delta} & c_{12} c_{23}-s_{12} s_{23} s_{13} e^{i \delta} & s_{23} c_{13} \\
s_{12} s_{23}-c_{12} c_{23} s_{13} e^{i \delta} & -c_{12} s_{23}-s_{12} c_{23} s_{13} e^{i \delta} & c_{23} c_{13}
\end{array}\right),
$$

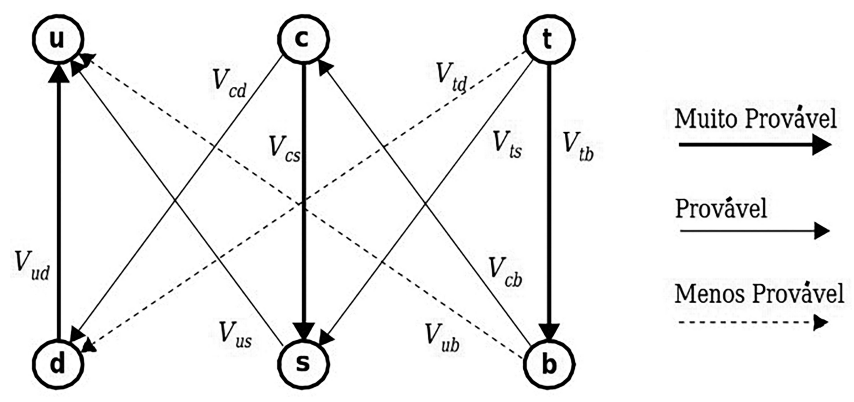

Figura 3: Ilustração das probabilidades de decaimentos dos quarks que é proporcional à $\left|V_{i j}\right|^{2}$. 
Onde $s_{i j}=\operatorname{sen}\left(\theta_{i j}\right), c_{i j}=\cos \left(\theta_{i j}\right)$, e $\delta$ é a fase (responsável última por toda a violação de $\mathrm{CP}$ em decaimentos de hádrons dentro MP). Os $\theta_{i j}$ são os ângulos de rotação $\theta_{12} \approx 13^{\circ}>\theta_{23} \approx 2.4^{\circ}>\theta_{13} \approx 0.22^{\circ}$, obtidos experimentalmente 3 . Podemos ver que os elementos em que há fase complexa estão multiplicados por $\operatorname{sen}\left(\theta_{i j}\right)$, que é pequeno. Isso ajuda a explicar o por quê da observação de VCP ser tão pequena (da ordem de $10^{-3}$ para o setor de charme, por exemplo). A parte imaginária de $V_{c d}, V_{c s}$ e $V_{t s}$ tem varios fatores $\operatorname{sen}\left(\theta_{i j}\right)$, o que faz com que esta seja muito pequena frente a parte real. Portanto, um aproximação razoável é de que apenas $V_{t d}$ e $V_{u b}$ são complexos.

\subsubsection{Parametrização de Wolfenstein}

Uma parametrização comum da matriz CKM, que considera medidas experimentais, é a de Wolfenstein, que explicita melhor a ordem de grandeza dos elementos $|V i j|$ [23] escritos em termos de A, $\lambda, \eta$ e $\rho$ que, por sua vez, estão relacionados com os termos de $s_{i j}$. Nesta parametrização, expande-se os elementos em ordens de $\lambda$, tendo a seguinte hierarquia: $\lambda=s_{12} \approx 0.22, s_{23} \approx \lambda^{2}$ e $s_{13} \approx \lambda^{3}$. De fato, em ordem $\lambda^{3}, V_{t d}$ e $V_{u b}$ são os únicos elementos que apresentam fase fraca, como visto antes:

$$
\begin{aligned}
V_{C K M} \approx & \left(\begin{array}{ccc}
1-\lambda^{2} & \lambda & A \lambda^{3}(\rho-i \eta) \\
-\lambda & 1-\lambda^{2} / 2 & A \lambda^{2} \\
A \lambda^{3}(1-\rho-i \eta) & -A \lambda^{2} & 1
\end{array}\right) \\
& +O\left(\lambda^{4}\right)
\end{aligned}
$$

onde $\mathrm{A} \approx 0.81, \rho \approx 0.13$ e $\eta \approx 0.34$ [3]. A condição de unitariedade da matriz de CKM estabelece que:

$$
\sum V_{i j} V_{i k}^{*}=\delta_{j k}
$$

Ou seja, a Eq. 16 se estende em 12 equações, sendo 6 delas identidades e 6 de ortogonalidade:

$$
\begin{array}{r}
V_{u d} V_{u b}^{*}+V_{c d} V_{c b}^{*}+V_{t d} V_{t b}^{*}=0 \\
\left|V_{u d}\right|^{2}+\left|V_{u s}\right|^{2}+\left|V_{u b}\right|^{2}=1 \\
V_{u s} V_{u b}^{*}+V_{c s} V_{c b}^{*}+V_{t s} V_{t b}^{*}=0 \\
\left|V_{c d}\right|^{2}+\left|V_{c s}\right|^{2}+\left|V_{c b}\right|^{2}=1 \\
V_{u d} V_{u s}^{*}+V_{c d} V_{c s}^{*}+V_{t d} V_{t s}^{*}=0 \\
\left|V_{t d}\right|^{2}+\left|V_{t s}\right|^{2}+\left|V_{t b}\right|^{2}=1 \\
V_{t d} V_{u d}^{*}+V_{t s} V_{u s}^{*}+V_{t b} V_{u b}^{*}=0 \\
\left|V_{u d}\right|^{2}+\left|V_{c d}\right|^{2}+\left|V_{t d}\right|^{2}=1 \\
V_{t d} V_{c d}^{*}+V_{t s} V_{c s}^{*}+V_{t b} V_{c b}^{*}=0 \\
\left|V_{u s}\right|^{2}+\left|V_{c s}\right|^{2}+\left|V_{t s}\right|^{2}=1 \\
V_{u d} V_{c d}^{*}+V_{u s} V_{c s}^{*}+V_{u b} V_{c b}^{*}=0 \\
\left|V_{u b}\right|^{2}+\left|V_{c b}\right|^{2}+\left|V_{t b}\right|^{2}=1
\end{array}
$$

Dividindo a Equação 17 por $V_{c d} V_{c b}^{*}$, pois já vimos que $V_{t d}$ e $V_{u b}$ são os termos complexos, tem-se:

$$
\frac{V_{u d} V_{u b}^{*}}{V_{c d} V_{c b}^{*}}+\frac{V_{t d} V_{t b}^{*}}{V_{c d} V_{c b}^{*}}+1=0
$$

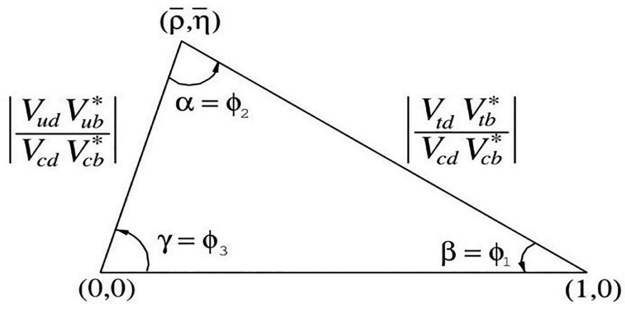

Figura 4: Ilustração do triângulo unitário [3].

E encontra-se um dos chamados triângulos unitários, visto na Figura 4 Como há 6 relações de ortogonalidade diferentes, há 6 triângulos diferentes com a mesma área $A=2 J_{C P}$, onde $J_{C P}$ é o parâmetro de Jarlskog [24]. Dentro do $\mathrm{MP}, J_{C P}$ é visto como a intensidade de violação de $\mathrm{CP}$, sendo $J_{C P} \approx 2,96 \times 10^{-5} 3$.

Os ângulos do triângulo unitário da Eq. 29 são assim definidos:

$$
\begin{gathered}
\beta=\phi_{1}=\arg \left(-\frac{V_{c d} V_{c b}^{*}}{V_{t d} V_{t b}^{*}}\right), \\
\alpha=\phi_{2}=\arg \left(-\frac{V_{t d} V_{t b}^{*}}{V_{u d} V_{u b}^{*}}\right), \\
\gamma=\phi_{3}=\arg \left(-\frac{V_{u d} V_{u b}^{*}}{V_{c d} V_{c b}^{*}}\right)
\end{gathered}
$$

\subsection{Assimetria de CP}

De uma forma geral, a fonte da violação de CP está vinculada a existência de termos complexos na densidade Lagrangeana, que são interpretados como fases em amplitudes de transição. Essas fases podem ser de dois tipos: CP-impar e CP-par. A primeira se trata de uma fase que muda de sinal em uma conjugação de $\mathrm{CP}$ portanto, ela é diferente para partícula e antipartícula enquanto a segunda não muda.

Dentro do MP, essas fases aparecem nas interações. Enquanto fases CP-impar podem aparecer via força fraca, as fases CP-par acontecem em interações fortes ou eletromagnéticas, por conta disso elas são renomeadas como fases fracas e fases fortes, respectivamente. A fase fraca, no MP, possui origem na matriz CKM, o que possibilita a previsão de quais tipos de decaimentos eventualmente apresentam VCP. Por sua vez, a fase forte possui duas possíveis fontes que estariam associadas aos chamados diagramas pinguim ou interações de estado final (re-espalhamento) 25].

Por simplicidade, vejamos um exemplo de um decaimento de uma partícula $P^{+} \rightarrow f$ e seu conjugado $P^{-} \rightarrow \bar{f}$, onde $f$ é um estado final possível, tal que este estado pode ser alcançado através de duas amplitudes (caminhos quânticos) diferentes. As amplitudes totais para $P^{+}$e $P^{-}$são:

$$
\begin{gathered}
A_{f}=\left|a_{1}\right| e^{i\left(\delta_{1}+\phi_{1}\right)}+\left|a_{2}\right| e^{i\left(\delta_{2}+\phi_{2}\right)} \\
\bar{A}_{\bar{f}}=\left|a_{1}\right| e^{i\left(\delta_{1}-\phi_{1}\right)}+\left|a_{2}\right| e^{i\left(\delta_{2}-\phi_{2}\right)},
\end{gathered}
$$


onde $\delta_{1,2}$ são as fases fortes, $\phi_{1,2}$ são as fases fracas e $a_{1,2}$ são amplitudes intermediárias. Fazendo a conjugação de $\mathrm{CP}$, a fase fraca troca de sinal enquanto a fase forte, não. A assimetria entre as taxas de decaimento pode ser quantificada pela grandeza $A_{C P}$, dada por:

$$
A_{C P}=\frac{\Gamma\left(P^{+} \rightarrow f^{+}\right)-\Gamma\left(P^{-} \rightarrow f^{-}\right)}{\Gamma\left(P^{+} \rightarrow f^{+}\right)+\Gamma\left(P^{-} \rightarrow f^{-}\right)}=\frac{\left|A_{f}\right|^{2}-\left|\bar{A}_{\bar{f}}\right|^{2}}{\left|A_{f}\right|^{2}+\left|\bar{A}_{\bar{f}}\right|^{2}} .
$$

Substituindo as Eqs. 33 e 34 na Eq. 35 encontramos:

$$
A_{c p}=\frac{2\left|a_{1} a_{2}\right| \operatorname{sen}\left(\delta_{2}-\delta_{1}\right) \operatorname{sen}\left(\phi_{2}-\phi_{1}\right)}{\left|a_{1}\right|^{2}+\left|a_{2}\right|^{2}+2\left|a_{1} a_{2}\right| \cos \left(\delta_{2}-\delta_{1}\right) \cos \left(\phi_{2}-\phi_{1}\right)} .
$$

Da Eq. 36 podemos ver que, uma vez que tenhamos interferência entre amplitudes com fases fortes e fracas diferentes, teremos uma assimetria de CP observável.

\section{Conclusão}

Neste trabalharam foram apresentados conceitos básicos de física de partículas e do Modelo Padrão. Nosso objetivo é avaliar, separadamente, a violação de CP nas interações fortes e fracas. Vimos que a violação de CP já foi observada nas interações fracas e existe possibilidade teórica de ser observada também nas interações fortes.

Apesar de não terem sido tratadas aqui, existem outras possíveis soluções para o problema da $\mathrm{CP}$ forte como quark $u$ sem massa e CP como uma simetria espontaneamente quebrada. No entanto, a solução mais convincente é mesmo a idéia de que o Modelo Padrão possui uma simetria adicional $U(1)_{P Q}$. Como vimos, essa solução prediz a existência de uma nova partícula. O áxion faria o papel de preencher o lugar de $\theta$, fazendo com que naturalmente ele seja 0 .

Para as interações fracas, tratamos sobre como é necessário existir pelo menos 3 gerações de quarks e léptons diferentes para que exista a mistura entre eles. É a partir dessa mistura que existe a possibilidade de violação de CP. Como vimos, esta já foi observada em mésons $K, B$ e $D^{0}$. O entendimento teórico do fenômeno se dá através da matriz CKM, que representa a possibilidade de transição entre quarks acontecer. Por fim, apresentamos a figura do $A_{C P}$, que desempenha um papel importante em observações de violação de CP.

\section{Referências}

[1] L. Canetti, M. Drewes e M. Shaposhnikov, New Journal of Physics 14, 095012 (2012).

[2] J.F. Donoghue, E. Golowich e B.R. Holstein, Dynamics of the Standard Model (Cambridge University Press, Cambridge, 2014), $2^{\text {a }}$ ed.

[3] Particle Data Group, P.A. Zyla, R.M. Barnett, J. Beringer, O. Dahl, D.A. Dwyer, D.E. Groom, C.-J. Lin, K.S. Lugovsky, E. Pianori, D.J. Robinson et al, Progress of Theoretical and Experimental Physics, 2020, 083C01 (2020).
[4] R. Aaij, C. Abellán Beteta, B. Adeva, M. Adinolfi, C.A. Aidala, Z. Ajaltouni, S. Akar, P. Albicocco, J. Albrecht, F. Alessio et al., Phys. Rev. Lett. 122, 211803 (2019).

[5] L.N.F. Ferreira, Estudos de sensibilidade para Violação de Carga-Paridade nos decaimentos $D^{+} \rightarrow K^{-} K^{+} \pi^{+}$ e $D^{+} \rightarrow \pi^{-} \pi^{+} \pi^{+}$no experimento LHCb. Dissertação de Mestrado, Pontifícia Universidade Católica do Rio de Janeiro, Rio de Janeiro (2021).

[6] A.E. Bernardini e S. De Leo, Revista Brasileira de Ensino de Física 27, 507 (2005).

[7] R. Milnitsky, Em Construção, 124 (2020).

[8] D. Possa, F. Pereira e J.A. Nogueira, Revista Brasileira de Ensino de Física 27, 407 (2005).

[9] J. Woithe, G.J. Wiener e F.F. Van der Veken, Physics Education 52, 034001 (2017).

[10] M.A. Moreira, Revista Brasileira de Ensino de Física 31, 1306 (2009).

[11] T.D. Lee e C.N. Yang, Phys. Rev. 104, 254 (1956).

[12] C.S. Wu, E. Ambler, R.W. Hayward, D.D. Hoppes e R.P. Hudson, Phys. Rev. 105, 1413 (1957).

[13] T.D. Lee, R. Oehme e C.N. Yang, Phys. Rev. 106, 340 (1957).

[14] J.P. Lees, V. Poireau, V. Tisserand, J. Garra Tico, E. Grauges, A. Palano, G. Eigen, B. Stugu, D.N. Brown, L. T. Kerth et al., Phys. Rev. Lett. 109, 211801 (2012).

[15] J.H. Christenson, J.W. Cronin, V.L. Fitch e R. Turlay, Phys. Rev. Lett. 13, 138 (1964).

[16] R. Lehnert, Symmetry 8, 114 (2016).

[17] J.L. Diaz-Cruz, W.G. Hollik e U.J. Saldana-Salazar, International Journal of Modern Physics A 33, 1850088 (2018).

[18] M.D. Schwartz Quantum Field Theory and the Standard Model (Cambridge University Press, Cambridge, 2014).

[19] A. Hook, arXiv:1812.02669 [hep-ph] (2018).

[20] F. Ostermann, Revista Brasileira de Ensino de Física 21, 415 (1999).

[21] S.L. Glashow, J. Iliopoulos e L. Maiani, Phys. Rev. D 2, 1285 (1970).

[22] M. Kobayashi e T. Maskawa, Progress of Theoretical Physics 49, 652 (1973).

[23] L. Wolfenstein, Phys. Rev. Lett. 51, 1945 (1983).

[24] C. Jarlskog, Phys. Rev. Lett. 55, 1039 (1985).

[25] I. Bediaga e C. Göbel, Progress in Particle and Nuclear Physics 114, 103808 (2020). 